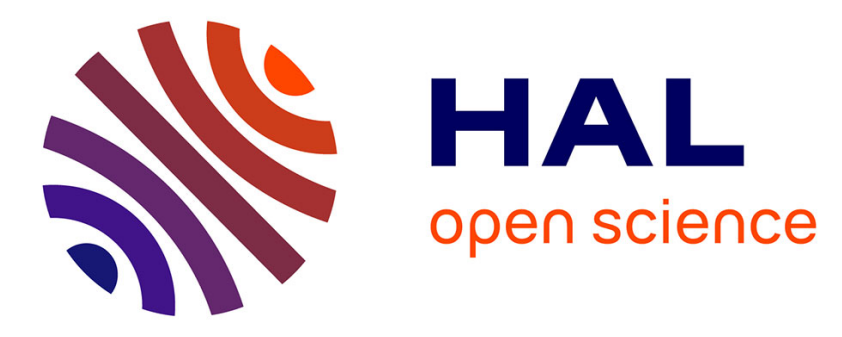

\title{
Plasmon induced enhancement of the electroluminescence signal of thin insulating polypropylene films
}

Kremena Makasheva, L. Montanari, Adriana Scarangella, G. Teyssedre

\section{To cite this version:}

Kremena Makasheva, L. Montanari, Adriana Scarangella, G. Teyssedre. Plasmon induced enhancement of the electroluminescence signal of thin insulating polypropylene films. 2020 IEEE 20th International Conference on Nanotechnology (IEEE-NANO), Jul 2020, Montreal (Virtual), Canada. pp.44-47, 10.1109/NANO47656.2020.9183435 . hal-02940243

\section{HAL Id: hal-02940243 \\ https://hal.science/hal-02940243}

Submitted on 22 Oct 2020

HAL is a multi-disciplinary open access archive for the deposit and dissemination of scientific research documents, whether they are published or not. The documents may come from teaching and research institutions in France or abroad, or from public or private research centers.
L'archive ouverte pluridisciplinaire HAL, est destinée au dépôt et à la diffusion de documents scientifiques de niveau recherche, publiés ou non, émanant des établissements d'enseignement et de recherche français ou étrangers, des laboratoires publics ou privés. 


\title{
Plasmon induced enhancement of the electroluminescence signal of thin insulating polypropylene films*
}

\author{
K. Makasheva, Member, IEEE, L. Montanari, \\ A. Scarangella, and G. Teyssedre, Senior Member, IEEE
}

\begin{abstract}
Chemical defects are of deleterious nature for the electrical properties of insulating polymers and can largely degrade their performance. Although these defects usually leave spectroscopic signatures in terms of characteristic luminescence peaks, it is nontrivial to assign, in an unambiguous way, the peaks to specific groups of defects because of the low intensity of the signal. In this work, we go beyond the traditional electroluminescence technique to record spectroscopic features of insulating polymers. By introducing a single plan of silver nanoparticles (AgNPs) at the near-surface of thin polypropylene films, the electroluminescent signal is enhanced by plasmon-related process. The latter leads much higher intensity and phasestabilization of the recorded spectra, thus improving the assignment of characteristic luminescence peaks.
\end{abstract}

\section{INTRODUCTION}

Insulating polymers are widely used in electrical engineering applications, such as high voltage direct current (HVDC) electrical cables, generators, transformers, capacitors, etc. [1]. However, their service life in systems is limited by the stresses (electrical, thermal, mechanical, etc.) they undergo. Knowledge on the response of insulation materials, and in particular insulating polymers to the applied electrical stresses is essential to determine their safety limits. These materials should sustain very high electric fields. For example, the typical service fields for HVDC cables are in the order of $20 \mathrm{kV} / \mathrm{mm}$. With time the ability of insulating polymers to sustain high electric fields decreases. It is mainly due to gradual degradation of the insulating materials and often leads to electrical breakdown. However, the degradation process takes certain time and is accompanied by modification in the response of the materials to the electrical stress. Moreover, the prebreakdown patterns may differ depending on the situation and the environmental factors (humidity, oxygen, radiation, corrosive agents, etc.) Electrical aging and degradation of insulating polymers therefore represent a constant interest in order to understand the fundamental mechanisms behind these effects, originating from evolving in time defects of the materials, and to contribute to the design and engineering of highly-performant electrical systems.

Electrical aging in insulators results from the induced thermal and higher energy chemical reactions after interaction of charged carriers with the dielectric material [2-4]. The aging of insulating polymers under electrical

*Research supported by the program IDEX Actions Thématiques Stratégiques - ATS 2015 of the Université de Toulouse under project SEPHIR (2016-066-CIF-D-DRVD). The authors acknowledge support from the UMS Raymond Castaing of the University of Toulouse and thank Mr Stéphane Le Blond du Plouy for the SEM observations. stress can partially be assigned to the space charges effect in the material. Different diagnostic methods are currently available to probe space charge, like wave propagation techniques (either pressure-wave -PIPWP- or pulsed electroacoustic -PEA- methods) or thermal methods (Laser Induced Modulation Method -LIMM- or Thermal Pulse - TP-) [5]. In the last few years, electrical modes derived from Atomic Force Microscopy (AFM), like Kelvin Force Microscopy (KPFM) [6, 7] and Electrostatic Force Distance Curve (EFDC) [8] advanced on the subject by revealing information on the charge injection and transport processes at nanoscale level. However, given the principle of operation, the above mentioned methods can reveal the presence of space charge but they are not sensitive to the energetics of charge carriers.

As demonstrated previously, the electroluminescence (EL) appears appropriate diagnostic tool to address the aging problem of insulating polymers because the degradation process involves energy dissipation in the chemical bond energy range (of a few eV) that corresponds to the UV-vis range of the optical spectrum [9-15]. EL is the excitation of luminescence as a result of the existence of an applied potential difference in the probed material. Basically the EL emission is a dynamic response of charges transport in the polymer material. The appearance of EL can be considered as a sign for the beginning of aging of polyolefin polymers under electrical stress. Spectroscopic analyses thus give indication on the nature of the transition process, i.e., on the dissociative versus non-dissociative excited states relaxation, defining in that way the energy decay mechanism: chemical versus physical one.

The EL of insulating polymers, such as polyethylene and polypropylene, is a specific response of the material which shows charge-structure interactions including the relaxation of kinetic energy by hot carriers, or modifications in their potential energy during radiative recombination of charges [11-15]. However, one of the challenges to meet when applying EL to probe aging mechanisms of polyolefin polymers is the low intensity of the obtained signal $[14,15]$. This problem is even more complex in the case of polyethylene and polypropylene, as these are weakly emitting materials under electrical field. As a result, the resolution of recorded spectra does not allow fine interpretation. To overcome this problem, we present in this paper a study on the possibility to analyze the

The authors are with LAPLACE laboratory, Université de Toulouse; CNRS, UPS, INPT; 118 route de Narbonne, F-31062 Toulouse, France (corresponding authors: kremena.makasheva@laplace.univ-tlse.fr and gilbert.teyssedre@laplace.univ-tlse.fr). 
spectroscopic signature of insulating polypropylene films by plasmon induced enhancement of their characteristic EL spectra.

\section{EXPERIMENTAL PART}

\section{A. Description of the structure and the plasma process}

The insulating polymer material under study was a thin bi-oriented polypropylene (BOPP) film (thickness of $17.8 \mu \mathrm{m})$. The BOPP film, provided by KOPAFILM, Germany, was with different roughness for the two faces.

A very thin nanocomposite layer, consisting of a single layer of silver nanoparticles (AgNPs) embedded in a very thin organosilicon (SiOC:H) matrix, was deposited on the side of larger roughness of the BOPP film by a plasma process. To perform the EL-measurements the structure was both sides covered by $100 \mathrm{~nm}$-thick ITO layers with $90 \%$ transparency and resistivity of $16 \times 10^{-3} \Omega / \mathrm{cm}^{2}$. A schematic cross-view of the structure is shown in Figure 1(a) and an optical image on top of the final sample is given on Figure 1(b).

(a)
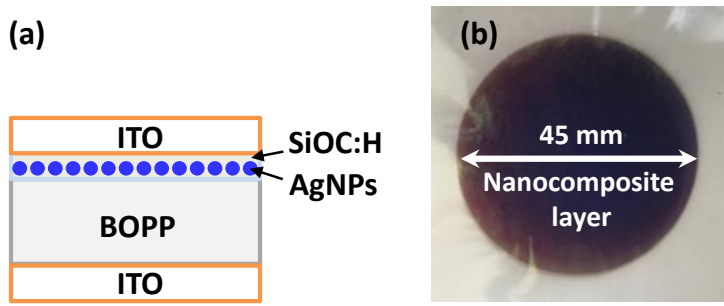

Figure 1. (a) Schematic of the studied structure in cross-section view and (b) Optical image of the final sample taken on the top.

The nanocomposite layer was deposited in two steps process: 1-plasma sputtering to create the layer of AgNPs and 2-plasma polymerization of hexamethyldisiloxane (HMDSO) precursor to cover the AgNPs by an organosilicon layer. The plasma deposition was performed in an axially-asymmetric capacitively-coupled RF $(f=13.56 \mathrm{MHz})$ discharge that successfully combines sputtering of a metal target and plasma polymerization. Details about the plasma process are given elsewhere [16]. The discharge powered electrode (smaller electrode) was Ag-made target to bear the sputtering. For the sputtering step the plasma was maintained in pure argon at low pressure $(p=5.4 \mathrm{~Pa})$ with RF power of $\mathrm{P}=20,40$ and $80 \mathrm{~W}$ $\left(\mathrm{V}_{\mathrm{dc}}=-546,-750\right.$ and $-1000 \mathrm{~V}$, respectively $)$. The sputtering time was fixed to $5 \mathrm{~s}$ or to $12 \mathrm{~s}$. $[16,17]$.

The very thin $\mathrm{SiOC}: \mathrm{H}$ cover layer was deposited in the same reactor after decomposition in the plasma of HMDSO at total pressure of $\mathrm{p}_{\mathrm{tot}}=6.6 \mathrm{~Pa}$. The power injected in the Ar-HMDSO plasma for this second step was $\mathrm{P}=80 \mathrm{~W}$ which induced a bias voltage of $\mathrm{V}_{\mathrm{dc}}=-900 \mathrm{~V}$.

\section{B. Structural and optical characterizations}

At each step of the elaboration process the samples were structurally and optically characterized. The size and surface density of AgNPs were determined after processing of Scanning Electron Microscopy (SEM) images. Optical transmittance spectra were recorded using a Hewlett Packard HP8452A Diode Array Spectrophotometer in the
UV-vis-NIR range $(190-820 \mathrm{~nm})$ to obtain the position of the AgNPs Local Surface Plasmon Resonance (LSPR). A Semilab 2000 spectroscopic ellipsometer was used to determine the thickness of the $\mathrm{SiOC}: \mathrm{H}$ cover layer.

\section{Electroluminescence characterization}

EL measurements [13-15] were achieved under secondary vacuum $\left(<10^{-6} \mathrm{mbar}\right)$ in order to avoid electrical discharges in the ambient. They were performed under ac voltage with variable frequency and electric field, up to $180 \mathrm{kV} / \mathrm{mm}$, using cooled detectors; being a photomultiplier in photon counting mode for EL-field intensity and phase patterns, and a CCD camera coupled to a dispersive system for spectra acquisition.

\section{RESULTS AND DISCUSSION}

\section{A. AgNPs Localized Surface Plasmon Resonance - LSPR}

The mean size of the obtained AgNPs is smaller than $10 \mathrm{~nm}$ for the lowest applied power of $20 \mathrm{~W}$ and of $15 \pm 10 \mathrm{~nm}$ and $20 \pm 10 \mathrm{~nm}$ for the $40 \mathrm{~W}$ and $80 \mathrm{~W}$, respectively. The AgNPs are organized in the plane in a single layer. Depending on the time of sputtering the size and density of AgNPs are different and the single plan can be discrete for short sputtering times $(5 \mathrm{~s})$ or percolated for longer sputtering times (12 s) at fixed power and pressure. The resultant organosilicon cover layer is conformal to the AgNPs. It is with thickness of only $6.5 \mathrm{~nm}$.
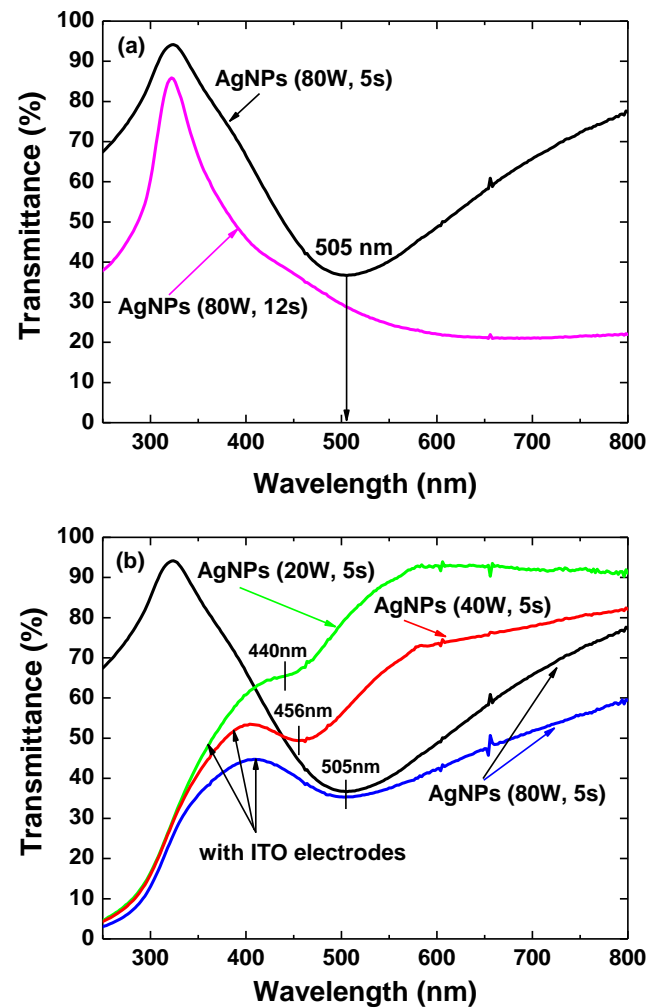

Figure 2. Transmittance spectra of the structures with AgNPs elaborated with different plasma powers and for different times: (a) Comparison between discrete $\left(\mathrm{P}=80 \mathrm{~W}, \mathrm{t}_{\mathrm{s}}=5 \mathrm{~s}\right)$ and persolated $\left(\mathrm{P}=80 \mathrm{~W}, \mathrm{t}_{\mathrm{s}}=12 \mathrm{~s}\right)$ AgNPs in terms of presence of LPSR of AgNPs and (b) Influence of the AgNPs size and ITO layers on the transmittance spectra. 
UV-vis-NIR transmission spectra of the elaborated samples containing AgNPs are presented in Figure 2. The spectra for layers of discrete and percolated AgNPs are shown in Figure 2(a). One observes the LSPR of AgNPs $\left(20 \pm 10 \mathrm{~nm}\right.$ size and surface density of $\left.2 \times 10^{11} \mathrm{NPs} / \mathrm{cm}^{-2}\right)$ of the discrete structure at $505 \mathrm{~nm}$. The LSPR disappears when the AgNPs are percolated in the layer. When elaborated with lower plasma power the AgNPs are smaller in size and their LSPR is blue shifted as shown in Figure 2(b). However, the AgNPs size variation gives the possibility to probe different chemical bonds in the BOPP structure. The influence of the conductive ITO electrodes is also shown in Figure 2(b). One can notice that the conductive ITO electrodes limit the analyses to the visible range, which is in accordance with the targeted energies.

\section{B. Enhancement of the electroluminescence intensity}

EL-intensities of bare BOPP sample and of the AgNPs/SiOC:H tailored one as function of the applied electric field are shown in Figure 3. One can clearly notice the very strong enhancement of the EL-signal in presence of AgNPs. Actually, the plasmon induced enhancement of the EL spectrum can be more than 3 orders of magnitude. Moreover, the detection threshold is much lower. Even with a field of $10 \mathrm{kV} / \mathrm{mm}$ the BOPP signature becomes readable. It was previously shown that switching form gold to ITO electrodes produced a decrease in the EL emission from BOPP along with a change in the shape of the EL-field characteristic in which the bump-like shape obtained for Au-electrodes disappears with ITO. This was explained by the contribution from surface plasmon (SP) emission in the red domain $[10,14,15]$ in case of gold; such emission being dominating at low ac field.

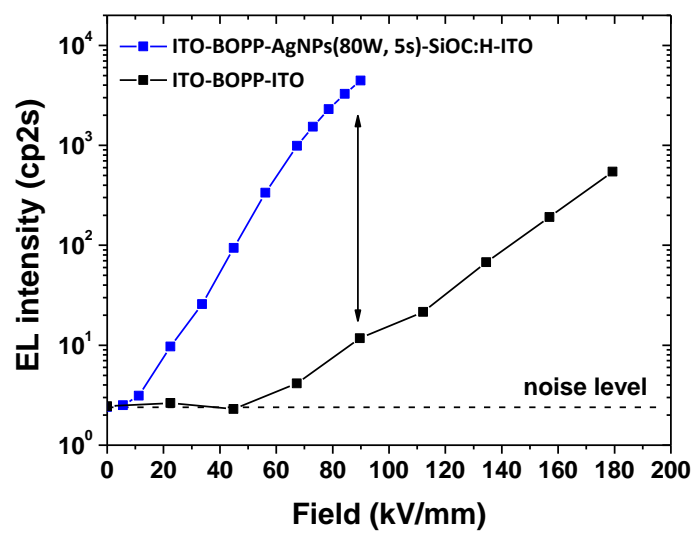

Figure 3. Electroluminescence vs. electric field under $50 \mathrm{~Hz}$ ac stress of bare and tailored BOPP films.

There are indeed different possibilities for the enhancement of the EL intensity: (i) stronger excitation, meaning larger number of recombination events; (ii) larger quantum yield (larger probability for radiative recombination); (iii) AgNPs contribution to the emission signal. To get further into the underlying process, complementary information is achieved through phaseresolved EL patterns and EL emission spectra.
Figure 4 compares the EL phase patterns obtained without and with the AgNPs/SiOC:H nanocomposite stack. Data are obtained at different applied electric fields owing to the strong difference in the EL yield (Figure 3). One can clearly see that there is no strong dependence of the shape of the pattern on the electric field and that the advance of phase is not largely pronounced in presence of AgNPs. This appears to be a quite distinct behavior compared to results obtained with Au electrodes where in general the advance of phase is larger when the SP emission is dominant [15].
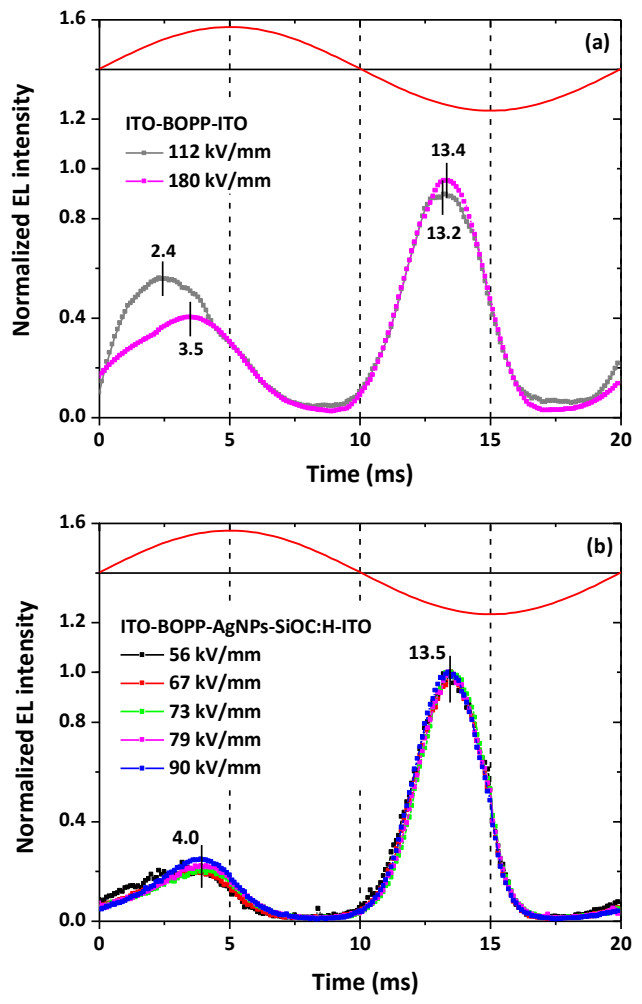

Figure 4. Phase-resolved EL patterns obtained on (a) BOPP and (b) one side tailored BOPP by an AgNPs/SiOC:H nanocomposite stack.

We have shown previously that the EL spectrum of BOPP, and more generally of polyolefins, can be deconvoluted into different contributions peaking at 415 , 505 and $570 \mathrm{~nm}$, all of these bands being characteristic of existing defects or created chemical functions into the material $[10,14,15]$. Combined with the absorbance spectrum of the AgNPs/SiOC:H tailored BOPP film (Figure 5) one can realize the strong coupling at the same energy ( $2.45 \mathrm{eV}$ or $\lambda=505 \mathrm{~nm}$ ) between the LSPR of the AgNPs and the contribution of recombination-induced luminescence involving conjugated $\mathrm{CC}$ bonds bound to $\mathrm{CO}$ species [14]. Moreover, the spectrum obtained for tailored with AgNPs BOPP films (Figure 5) reveals the presence of an additional band at about $650 \mathrm{~nm}$ that does not appear in the spectra obtained on bare BOPP (not shown here). In case of Au-electrodes an extra broad emission at 700$750 \mathrm{~nm}$ was attributed to the SP emission [14]. The additional band at $650 \mathrm{~nm}$ can be attributed to the SP emission from the AgNPs in SiOC:H environment. 


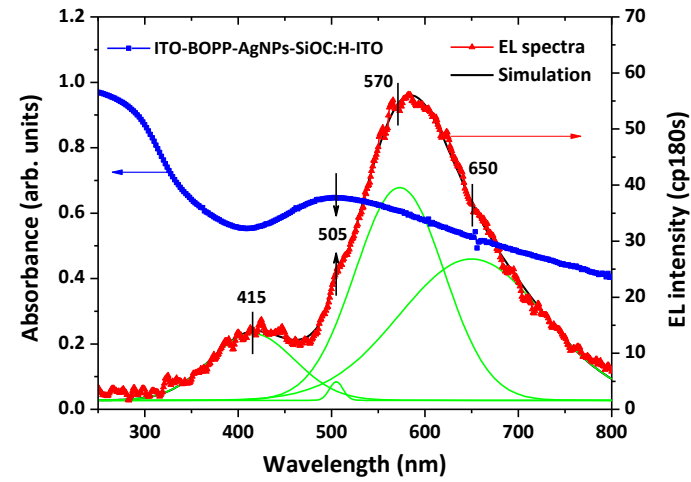

Figure 5. Absorbance and ac-EL spectrum of an one side tailored BOPP by an AgNPs/SiOC:H nanocomposite stack. The EL spectrum is obtained with an ac crest field of $90 \mathrm{kV} / \mathrm{mm}$. The spectrum is deconvoluted into the sum of different Gaussian functions with peak wavelength indicated in the figure.

However, this does not constitute the only explanation to light enhancement. Other possibilities are to be considered. Firstly, the AgNPs/SiOC:H layer may favor charge injection, and then charge trapping on the AgNPs. The number of recombination events might be amplified by combining the enhanced charge injection efficiency and the capability of AgNPs to stabilize injected charges [16]. Second, a sensitization of the PP due to the presence of AgNPs may occur, either through the formation of oxidized groups in the AgNPs environment, or through metal enhanced luminescence processes, that involve the LSPR in presence of metallic nanoparticles. This later process, complex indeed, has been recently reported in different nanoscience fields $[18,19]$.

\section{CONCLUSION}

Evidences on the possibility to change energetics of the charge carriers near the interface is brought by electroluminescence measurements on tailored by AgNPs interfaces of capacitor films. Not only the light intensity is found much stronger in presence of AgNPs, but the detection of light emission starts at lower field. Such strong emission is most likely due to a radiative surface plasmon relaxation from the AgNPs in organosilicon environment. Depending on the size and density of AgNPs one can obtain different levels of energy absorption at the LSPR, thus providing flexibility for further enhancement of the response, according to probed material. Further work will be directed to explore the metal enhanced electroluminescence process by considering the localized surface plasmon resonance in presence of AgNPs in order to probe structural modifications and subsequent aging of polymeric insulations.

\section{ACKNOWLEDGMENT}

The authors acknowledge fruitful discussions with Dr. Christian Laurent.

\section{REFERENCES}

[1] L. A. Dissado and J. C. Fothergill, Electrical Degradation and Breakdown in Polymers. Peter Peregrinus Ltd., London, 1992.

[2] L. Sanche, "Nanoscopic aspects of electronic aging in dielectrics," IEEE Trans. Dielectr. Electr. Insul., vol. 4, pp. 507-543, 1997.

[3] G. Teyssedre and C. Laurent, "Charge transport modeling in insulating polymers: from molecular to macroscopic scale," IEEE Trans. Dielectr. Electr. Insul., vol. 12, pp. 857-875, 2005.

[4] G. Mazzanti, G. C. Montanari, and L. A. Dissado, "Electrical aging and life models: the role of space charge," IEEE Trans. Dielectr. Electr. Insul., vol. 12, pp. 876-890, 2005.

[5] R. J. Fleming, "Space charge profile measurement techniques: recent advances and future directions", IEEE Trans. Dielectr. Electr. Insul., vol. 12, p. 967, 2005.

[6] E. Palleau, L. Ressier, Ł. Borowik, and T. Mélin, "Numerical simulations for a quantitative analysis of AFM electrostatic nanopatterning on PMMA by Kelvin force microscopy," Nanotechnology, vol. 21, p. 225706, 2010.

[7] C. Villeneuve-Faure, L. Boudou, K. Makasheva and G. Teyssedre, "Methodology for extraction of space charge density profiles at nanoscale from Kelvin probe force microscopy measurements," Nanotechnology, vol. 28, p. 505701, 2017.

[8] C. Villeneuve-Faure, L. Boudou, K. Makasheva, and G Teyssedre, "Towards 3D charge localization by a method derived from atomic force microscopy: the electrostatic force distance curve," J. Phys. D: Appl. Phys., vol. 47, p. 455302, 2014

[9] G. Teyssedre, L. Cissé, D. Mary, and C. Laurent, "Identification of the components of the electroluminescence spectrum of PE excited in uniform fields," IEEE Trans. Dielectr. Electr. Insul., vol. 6, p. 11, 1999.

[10] G. Teyssedre, G. Tardieu, D. Mary, and C. Laurent, "Ac and dc electroluminescence in insulating polymers and implication for electrical ageing," J. Phys. Appl. Phys., vol. 34, pp. 2220-2229, 2001.

[11] C. Laurent and G. Teyssedre, "Hot electron and partial-discharge induced ageing of polymers," Nuclear Instruments and Methods in Physics Research B, vol. 208, pp. 442-447, 2003.

[12] G.-J. Zhang, K. Yang, M. Dong, W.-B. Zhao, and Z. Yan, "Surface electroluminescence phenomena correlated with trapping parameters of insulating polymers," App. Surf. Sci., vol. 254, pp. 1450-1455, 2007.

[13] G. Teyssedre and C. Laurent, "Evidence of hot electron-induced chemical degradation electroluminescence spectra of polyethylene," J. Appl. Phys., vol. 103, p. 046107, 2008.

[14] B. Qiao, G. Teyssedre, and C. Laurent, "Electroluminescence and cathodoluminescence from polyethylene and polypropylene films: Spectra reconstruction from elementary components and underlying mechanisms," J. Appl. Phys., vol. 119, p. 024103, 2016.

[15] B. Qiao, C. Laurent, and G. Teyssedre, "Evidence of Exciton Formation in Thin Polypropylene Films under AC and DC Fields and Relationship to Electrical Degradation," IEEJ Trans. Fundam. Mater., vol. 136, pp. 74-80, 2016.

[16] K. Makasheva, C. Villeneuve-Faure, C. Bonafos, C. Laurent, A Pugliara, B. Despax, L. Boudou, and G. Teyssedre, "Dielectric engineering of nanostructured layers to control the transport of injected charges in thin dielectrics," IEEE TNANO, vol. 15, pp. 839848, 2016.

[17] L. Milliere, K. Makasheva, C. Laurent, B. Despax, L. Boudou, and G. Teyssedre, "Silver nanoparticles as a key feature of a plasma polymer composite layer in mitigation of charge injection into polyethylene under dc stress," J. Phys. D: Appl. Phys., vol. 49, p. 015304, 2016.

[18] Y. Jeong, Y.-M. Kook, K. Lee, and W.-G. Koh, "Metal enhanced fluorescence (MEF) for biosensors: General approaches and a review of recent developments," Biosens. Bioelectron., vol. 111, pp. 102-116, 2018

[19] A. Scarangella, M. Soumbo, A. Mlayah, C. Bonafos, M.-C. Monje, C. Roques, C. Marcelot, N. Large, T. Dammak, and K. Makasheva, "Detection of the conformational changes of Discosoma red fluorescent proteins adhered on silver nanoparticles-based nanocomposites via surface-enhanced Raman scattering,' Nanotechnology, vol. 30, p. 165101, 2019. 